\title{
The Usage of Sentiment Analysis for Hearing the Voice of the Customer and Improving Businesses
}

MSc. Majlinda Axhiu

International University of Struga, m.axhiu@eust.edu.mk

PhD. Florida Veljanoska

International University of Struga, f.veljanoska@eust.edu.mk

PhD. Biljana Ciglovska

International University of Struga, b.ciglovska@eust.edu.mk

MSc. Mirlinda Husejni

Doi:10.5901/jesr.2014.v4n4p401

University of Wales Trinity Saint David mirlinda.husejni@gmail.com

\section{Abstract}

Thanks to social media, organizations now have the opportunity to continuously monitor customer feedback, laws regulations and policies. The voice of the customer has become more influential than ever before, because people make sure that whatever they say is heard by their friends and followers in microblogs. However, information gathered from social media has little value unless it is examined against other facts and used to forecast trends and risks. Considering the great importance of customer opinions, business stakeholders mostly care about the processing time and accuracy of big data, which is now easily found. That is why, for opinion processing we are suggesting Sentiment Analysis, which is a set of methods, that perceive, measure, report and utilize attitudes, opinions, and emotions automatically. Our aim is to show that the engagement of Sentiment Analysis in business can be used as an alert for the key stakeholders, to be informed for specific positive or negative discussions and issues which may affect the brand. From the case studies that we have analyzed about big and medium companies, we have found out that by measuring the customer's perception of different goods, services and commercials, Sentiment Analysis has helped on improving their products, operations and department communications. It has even reached a great impact on intangible assets of the companies. The main contribution of this paper comprises of the usage of Sentiment Analysis in companies, in a manner that they can improve their business and reach more potential customers and business values, as well as to become a good reference point for further researches in this field.

\section{Introduction}

Sentiment analysis is a set of methods, usually implemented in computer software, that perceive, measure, report and utilize attitudes, opinions, and emotions, which generally are called sentiments and can be found in online, social, and enterprise information sources.

Once it has been a sleepy area of computing, which now is no longer dormant. It has been around in computer science circles for some time, but is progressively becoming more sophisticated and therefore useful. The rise and proliferation of social media and social networks such as Facebook and Twitter, has fueled major interest in this field and a growth in tools to respond to the demand.

Customer conversations and reviews are taking place 24/7. Thanks to social media, organizations now have the opportunity to continuously monitor customer feedback in real time, laws regulations and policies. Information gathered from social media has little value unless it is examined against other facts, applied to changing models and used to forecast trends and risks. The sentiment analysis tools are united in using technology to monitor opinions and gather qualitative insights, measure them and report what is found in a palatable form. The main focus is on the specific 
application of classifying reviews as to their polarity (either positive or negative).

The most attractive sources of data that business stakeholders want to use are microblogs. Microblogs like Twitter, on which users post real time reactions and opinions about "everything", poses newer and different challenges. People make sure that whatever they say is heard by their friends and followers, so they have become more influential than ever before.

Researchers inquire about sentiment at various linguistic levels, such as: words, phrases, sentences and documents. These tasks analyze sentiment at a fine-grained level and can be used to improve the effectiveness of a sentiment classification.

To automate sentiment analysis, different approaches have been applied to predict the sentiment of words, expressions or documents. There are Natural Language Processing (NLP) and pattern-based, machine learning algorithms such as Naïve Bayes, Maximum Entropy and Support Vector Machine. (Abbasi, Chen \& Salem 2008)

Opinion mining is not just about analyzing social media conversations but it is about any information source that captures subjective information. It can involve social conversation and also direct and indirect feedbacks, online news, presentations, even scientific papers. (Grimes 2011)

Sentiment analysis lets marketers and market researchers, customer service and support staff, product managers, etc. get at root causes, at explanations of behaviors that are captured in transaction and tracking records.

It means better targeted marketing, faster detection of opportunities and threats, brand-reputation protection, and the ultimate aim, profit.

\section{Social Media Presence}

Taking a look at the way of communication that occurs within an organization and outside of it- including all stakeholders, historically, there are radical differences.

Starting with emails, of which the basic idea has remained essentially unchanged since the first email was sent, which is really good for one-on-one communication, then passing to instant messaging, with which are allowed real-time communication, and ending to social media.

Nowadays there are a lot of changes in the way that businesses approach employees' social media use. Not long ago chatting or tweeting in office hours was prohibited. Lots of companies have even been cracking down on what employees have posted on personal Facebook pages in their free time. However, all the researches have shown that forbidding employees to use social media, at the end of the day has just resulted into bad business. Lately, some big organizations have gotten the message and started to be more active on this field.

According to the statistics that MarketTools provided at the end of 2011, 23\% of companies offered customer service via Facebook and 12\% offered customer service via Twitter. However, the total percentage of $35 \%$ differs a lot from the percentage that is shown in the statistics at the end of 2012.

The top users understand that social media is a conversation between their company and their stakeholders, not a monologue. More effective companies use social media to interact with customers by creating online customer groups, including review sites, multi-media sharing, blogs and discussion forums.

Trying to learn more for the benefits of usage of social media, a survey is conducted in which there have been asked the effective users and the ineffective ones. Below is shown the graph of the answers and as it is seen the major effort (50\%) is related to the increase of awareness of the organization or brand.

The main point that we should be concerned about is that the opinion of effective users have encapsulated the main object of the sentiment analysis research. 


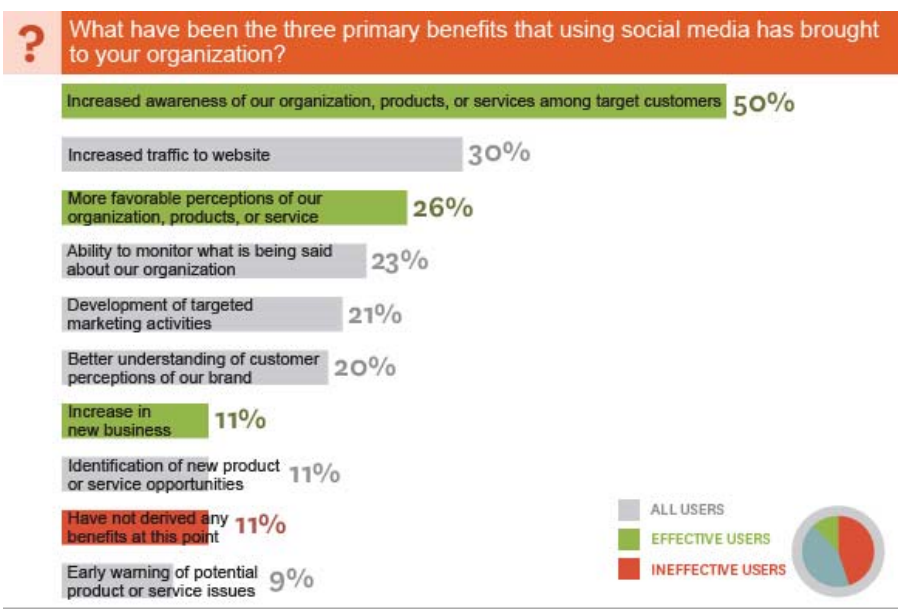

Figure 1: Benefits of Social Media At: http://hbr.org/web/slideshows/social-media-what-most-companies-dont-know/1slide

A large percentage of organizations still hesitate to get serious about social media and as a result they cannot see the benefits that they can get. The toughest challenges for executives entail tying social investments back to the outcomemeasuring its effectiveness, involving social media efforts to ROI and understanding the real difference that social efforts make to the business. Although they are aware of how social media can be a powerful tool, most of them still aren't sure enough.

While on the other hand for the effective users it is clear now, that beside the old methods of marketing with the usage of social media they can better promote their brand, monitor trends among customers, learn the opinion and perceptions of the products and services, increasing the value of the business, and even research new product ideas.

Taking into consideration all of the above mentioned possible advantages, experts support the idea that companies should hire special staff dedicated to social media activities. However, only $12 \%$ of the companies have done it. In most of the companies social media has also trouble in getting a piece of the budget pie. Only $20 \%$ of social-media-using companies have dedicated a part of the budget to it.

The reason why these percentages should rise has to do with the strong belief that social media can change the culture within an organization and increase the outcomes in unexpected ways.

First of all it helps companies to hire better people. If the job openings are published in social media, the entire process will be public, transparent and available for everyone to see. This may represent a labour market where all job hunters and staff are together. Through social media, companies get to know candidates on a more personal level and sometimes this can be very useful.

Secondly, social media can help in breaking down the hierarchies in the workplace, by closing the gap between management and operation level. The managers now give the freedom to anyone including the employees and interns to tweet them as well as other department heads at any time. Twitter, with its conciseness and informality, makes possible for the managers to communicate with hundreds of their employees, in a way that would be unimaginable on email or in person.

At last, social media empowers employees to become brand missioners. The final outcomes of this king of social engagement are really huge. When the employees share their experiences all the time on social networks and they blog about their work, they reach an influential audience of colleagues, peers and potential customers. It is important that all of this communication is a two-way benefit: employees are able to build their individual brand and on the other hand cultivating the company brand at the same time. Taking as an example if a company has 30 employees, and each of them has 200 followers, then altogether the brand of the company is spread to 6,000 people.

That is why social media should not be considered as a threat; on the contrary it is a way to improve internal communication within the company, seek out better hires and turn employees into brand ambassadors.

This statement also is supported by McKinsey, when in its recent report (2012) is shown that more effective use of social technologies could release $\$ 1.3$ trillion in value for businesses.

Below are discussed some examples about the companies of different categories and their usage of social media. 
According to some research that "Hotelmarketing" has done, $62 \%$ of customers are looking for more support through social media. So, the brand reputation and promotions depend on how companies use social media.

However the gap between the customers' desires and what companies emphasize is in fact growing. This happens because not many destinations or destination management organizations try to use their online presence to support the needs of their customers, or potential customers. They are occupied mostly by promoting the qualities of their goods and don't have time to ask what their customers want. And more importantly to answer to the customer questions and offer quality, individualized advice.

Likely this passiveness also is present in Health Care institutions, because only $9 \%$ of customers are using social media to contact hospitals.

On the other hand there are a number of big companies that use social media in massive way. Last year, $73 \%$ of Fortune 500 organizations were active on Twitter, while more than $80 \%$ of executive managers believed that the engagement of social media led to increased sales.

More precisely, the sale departments use social media to generate leads and track customers as they move through the sales channel. Human Resources departments are using social media to connect with job seekers and simplify the application process. Operations and distribution teams estimate supply chains, while research and development group brainstorm product ideas.

Taking the data from social media and using them in real-time analysis through sentiment analysis tools can be very useful for business decisions. These tools have already being used by eminent companies such as Nestle for following customer sentiment, Wall Street to predict stock prices, GE to speed up repairs to the electrical grid and TMobile to prevent customer defections. As it is seen the usage of social media is not limited and can be helpful for any sector; such as food and healthcare, pharmaceutical, governmental, electrical, engineering, etc.

\section{Benefits of Sentiment Analysis in Businesses}

Taking into consideration that reaching the big data is very easy in this age, by using sentiment analysis, the business owners can easily find out how the public is feeling about certain things.

It is very important to know the specific goal of gathering data, for instance if they will help us to understand how people feel about our brand, or they will help on understanding an emerging problem concerning a product or service from our company. So, there are a lot of reasons why the collected data may be needed and analyzed, but we need to specify beforehand so that the gathered data are inline with the expected outcome.

By measuring the customer's perception of different goods, services and commercials, businesses can use sentiment analysis to improve their products, their operations, departments, and even to have a great impact on intangible assets of the companies.

Advertising companies and businesses use sentiment analysis also to discover exclusive strategies for their advertising materials and advertising campaigns.

So, the general purpose is to gain the trust of the consumers, which will result in business growth and expansion. That is why the business owners should take care of customer's issues also. It is very common for a consumer to write a bad viewpoint about a specific product, service or brand, in online communities. It is very important for the companies to detect these letdowns and take appropriate actions. They should get in touch with the consumers and solve their concern at the earliest.

By creating a sense of respect among consumers, social media and sentiment analysis have a vital importance for the growth of an organization. The consumers will believe in the brand and will be convinced about the quality of the products and services.

Another benefit from tracking the positive and negative points of the business in real time (24/7) is the detection of urgent threats to reputation, as well as to benchmark the performance of the company against its competitors.

The engagement of sentiment analysis in business can be used as an alert for the key stakeholders to be informed of specific positive or negative discussions and issues which may affect the brand. By this the companies will have a continuous control of their brand reputation.

\section{Case Studies}

\subsection{Burger King Smoothies}

Since there were a lot of competitors for Smoothies, Burger King decided to use sentiment analysis applications and to 
see how people like this product.

The methodology that they used included the process of collecting opinions, cleaning them, categorizing and calibrating them. So, first of all they searched the Internet for relevant opinions and messages, and then cleaned them out of spam and non-relevant comments. After that all the data was categorized into different content areas, for instance pricing, flavor, commercials, etc. And at last their sentiment was calibrated into 5-point Likert scale.

During a search that was done in 2012 for each month separately considering the question "Where to find Smoothies?" resulted that firstly the main actors were Smoothie King and McDonald's, whereas Burger King had just a tiny slice of the pie. But, the situation changed drastically just after few months, where the main actor turned to be Burger King.

This situation is also shown in the following graph where it is shown a great increase at that period of time.

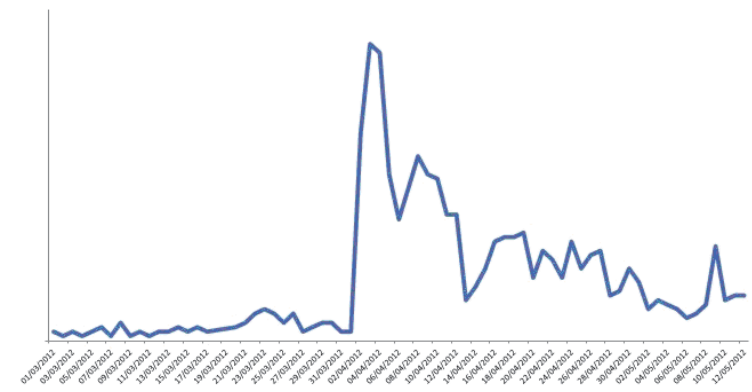

Figure 2: The fame of Burger King Smoothies At: http://www.slideshare.net/ResearchNow/conversition-august-webinarcan-cheeseburgers-and-fruit-smoothies-coexist

There were several categories in which were analyzed the opinions for Burger King's Smoothies, including the taste, healthy ingredients, ice and creativity.

Regarding the taste, people found the smoothies really tasty, which resulted in $67 \%$ of positive opinions. All of the positive comments which were much more in number stated that can be felt the real taste of fruits. On the other hand, the customers also found them very healthy and most of the comments were positive also in comparison with the Smoothies from other places.

Beside the positive aspects there were also some complains. The company found out that people complain about the ice, declaring that the smoothies are watered down or that the ice pieces are very big. So, for a great number of people smoothies were defined as "chunks of ice with some flavoring".

Except this issue a considerable number of people remarked that Burger King had a lack of creativity and was just copying McDonald's, so their smoothies were copycat. However, from the research it can be seen that people in fact liked the fact that Burger King served the same smoothies as McDonald's and they were asking for them.

In overall, the usage of sentiment analysis had a lot of benefits for the company, because beside the positive things that they heard about their product they also learned about the issue of ice and about copying one of its competitors. Even though at first sight the negative comments were a little bit disappointing for the company, it is good that they learned about these problems, which is of great importance.

Now, it was on their hands to improve their product and to solve the issue with the ice. On the other side, they also learned that copying the main competitor is not always a bad thing, and it can have a positive influence on the product, because a lot of people asked for smoothies just because they were the same as those from McDonald's.

\subsection{Big Mac vs. Whopper}

Another good point of sentiment analysis is that besides using it for measuring the overall sentiment toward a brand, it can be used also for comparative analyses among several products, services or brands.

This case study is conducted among two great competitors. It is done a detailed analysis for the McDonald's Big Mac and the Burger King Whopper.

For this evaluation are used thousands of comments and messages, which are gathered from different websites, including blogs, forums, questionnaires, photos, videos, etc. 
The data collected is a random sample and is valid for the period Nov. 1, 2010 until Jan. 31, 2011.

This meticulous analysis is achieved by social media research and combining content analysis. So, both hamburgers are evaluated piece by piece, starting from the bread and ending to the sauces.

Regarding the bun of the hamburgers, there were much more positive conversations for the Whopper $(30.5 \%)$ compared to Big Mac (20\%). The negative comments are with less difference, however still the percentage of Big Mac $(13.6 \%)$ is greater than the one of Whopper (10.7\%).

The other main ingredient of the hamburgers is the meat. The hamburger patty of the Big Mac is discussed much more, and respectively the positive sentiments (22.6\%) are more in number than those of Whopper (19.2\%).

Comments about tomatoes are more frequent for Whopper which has tomato, than Big Mac which does not have. Considering the fact that in Big Mac you may have tomato only when you ask for it, the comments for Whopper are far more positive.

On the other hand the lettuce on Big Mac gains more positive sentiments (22.9\%) than the lettuce on Whopper (15.4). Disproportionally the negative comments of Whopper's lettuce are almost doubled in number, compared to the amount of negative comments of Big Mac.

Considering the other ingredients, as it can be seen from the graph, the pickles of Big Mac generate more positive comments than the pickles of Whopper. However, the Big Mac pickles also collect more negative sentiments.

Even though with a slight difference, again Big Mac wins the battle against Whopper in thanks to onion.

Taking a look at the cheese of both burgers, we can easily notice the similar percentages of positive mentions; however the percentage of negative comments for Whopper's cheese is greater than Big Mac.

Passing to the ketchup, Big Mac does not include it and that is why it's positive percentage is only 17.7 which is almost half of the percentage of Whopper (34\%). The shortage of the ketchup in Big Mac is the main reason for the negative sentiments as well.

Considering the fact that Whopper does not have a unique sauce, there was a very small discussion about it (only $0.1 \%$ ) that is why here the main focus is on the Big Mac sauce, where the positive sentiments has reached up to $36.8 \%$.

Opposite to this, the main actor in the discussion of mayonnaise has been Whopper, since Big Mac doesn't include it (same as the ketchup). So, if a customer wants to eat his Big Mac with mayonnaise, he should ask for it. As a result the positive sentiments of Whopper were about $40.4 \%$ while those of Big Mac were around $37.5 \%$.

By all these partial evaluations which were done for all ingredients of the hamburgers, with a higher percentage of positive ratings and a lower percentage of negative ratings, the winning burger has been Whopper.

The overall parentages for the positive, negative and neutral sentiments for both burgers are shown in the figure below.

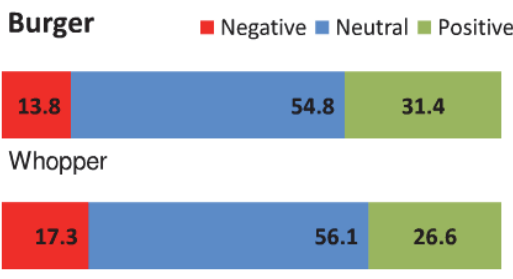

Big Mac

Figure 3: The overall sentiment about Whopper and Big Mac

Both companies were satisfied for entering this analysis, because now they were aware of their positive sides and of their weaknesses. Now, they could use all of this information and try to improve themselves, to gain competitive advantage and why not to enter in a new battle by using sentiment analysis.

\section{Conclusion}

In this paper it is taken a look at sentiment analysis and the classification of user opinions. Considering the great importance of customer opinions and the influence that they may have in business companies, the processing time and accuracy are the most significant factors. That is why in the automated classification, each opinion should be transformed into formal language and structured in a group of five elements, which will be understood by the machine. After that it is a 
must for the opinions to pass through the five steps of sentiment analysis in order to gain the final results.

Considering the success stories of the big and medium companies, we have seen that the gained business values are really great; starting from customer relationship management, the communication among different departments within an organization, the voice of employees, the advantages in advertising, brand reputation, etc. and ending into the final goal- business profit.

Taking into account all these values the business world has reached into a level where companies are ready even to invest in sentiment analysis and take its advantages.

Even though there are tons of benefits there are still a lot of possibilities for improvements. The first thing that should be considered in future works is the possibility to change the existing algorithms or reengineer them for reaching higher accuracy. Here should be taken into account all the challenges of understanding natural language, which are increasing each day.

Another field that should be worked on is multi-language systems, because until now the most used language in sentiment analysis systems is English; however there are huge corpuses of data that may be used also in other languages. This possibility is really important especially for medium and small businesses which may function locally in countries where the first language is not English.

At last, regarding our country and region we would say that there is needed a great work to be done, in order to awaken business people to see all the advantages that sentiment analysis may bring into their companies, and to stimulate them for implementing it.

\section{References}

Delo, C. 2011. Twitter Now Has 100 Million Active Monthly Users: CEO. [online] Available at: http://adage.com/article/digital/twitter-100million-active-monthly-users-ceo/229688/.

Delua, J. 2011. Big Data Meets Sentiment Analysis!. informatica, [blog] 27th June, Available at: http://blogs.informatica.com/perspectives /2011/06/27/big-data-meets-sentiment-analysis/

Deshp, E, M. and Sarkar, A. 2010. BI and Sentiment Analysis. 15 (2), p. 41.

Go, A., Huang, L. and Bhayani, R. 2009. Twitter sentiment analysis. Entropy, 17.

Grimes, S. 2008. Sentiment Analysis: Opportunities and Challenges. [online] Available at: http://www.b-eye-network.com/view/6744

Hbr.org. 2011. Social Media: What Most Companies Don't Know. [online] Available at: http://hbr.org/web/slideshows/social-media-whatmost-companies-dont-know/4-slide

Horn, C. 2010. Analysis and Classification of Twitter messages.

Hornor, T. n.d. Why Sentiment Analysis Is Essential for Small Businesses | Graziadio Voice: Business School Blog. [online] Available at: http://bschool.pepperdine.edu/studentblog/index.php/why-sentiment-analysis-is-essential-for-small-businesses/

King, R. 2011. Sentiment Analysis Gives Companies Insight Into Consumer Opinion. [online] Available at: http://www.businessweek.com/stories/2011-03-01/sentiment-analysis-gives-companies-insight-into-consumeropinionbusinessweek-business-news-stock-market-and-financial-advice

Markettools.com. 2011. Nearly One-Fourth of Companies Now Provide Customer Service via Facebook | MarketTools. [online] Available at: http://www.markettools.com/company/news-events/press-releases/nearly-one-fourth-of-companies-provide-customer-servicevia-facebook

Mashable. 2010. How Companies Can Use Sentiment Analysis to Improve Their Business. [online] Available at: http://mashable.com /2010/04/19/sentiment-analysis/

Pak, A. and Paroubek, P. 2010. Twitter as a Corpus for Sentiment Analysis and Opinion Mining.

Slideshare.net. 2012. Can Cheeseburgers and Fruit Smoothies Co-Exist?. [online] Available at: http://www.slideshare.net/ResearchNow /conversition-august-webinar-can-cheeseburgers-and-fruit-smoothiescoexist?utm_source=slideshow03\&utm_medium=ssemail\&utm_campaign=share_slideshow

Socialmention.com. 2013. Real Time Search - Social Mention. [online] Available at: http://www.socialmention.com/ 